\title{
Resecting the Lesion Combined with Local Flap Repairing for the Treatment of Infected Congenital Preauricular Fistula
}

\author{
Yuanming Jiang* \\ Ting $\mathrm{He}^{*}$ \\ Wei Liu
}

Department of Otolaryngology, Wuhan No. I Hospital, Wuhan, Hubei, 430022. People's Republic of China

*These authors contributed equally to this work
Correspondence: Yuanming jiang Department of Otolaryngology, Wuhan No. I Hospital, Wuhan, Hubei, 430022. People's Republic of China Tel +86-27-8533268I

Email jiangmingent@I26.com
Purpose: Evaluating the treatment of infected preauricular fistulas by resecting the lesion combining with local flap repairing.

Patients and Methods: This clinical study was implemented in Ningbo No. 1 Hospital and Wuhan No. 1 Hospital of China. We included 100 cases who were diagnosed with infected preauricular fistulas and needed further surgery treatment. Among them, 50 patients experienced the conventional treatment including a fully infection control following with a surgery to remove preauricular fistulas (conventional treatment group). Other 50 patients only took some simple pre-operation treatment for about 1-7 days, and then preauricular fistulas resection combining local flap repairing were performed regardless of the infection (local flap repairing group). The duration of total treatment procedure, healing index, and total cost were observed.

Results: All patients were discharged at about 7 days after surgery, the duration of the postoperative period had no significant difference between groups. The total duration of treatment (from any first treatment time point to patient discharge) was only $13.98 \pm 2.14$ days in the local flap treatment group compared with that of $43.06 \pm 8.24$ days in the conventional treatment group. Further, the total cost of treatment per patient in the local flap repairing treatment group was about $47.1 \%$ of that in the conventional treatment group.

Conclusion: For the treatment of infected preauricular fistula, performing a lesion removing surgery combining a local flap repairing can shorten the total treatment course, relieve patient's suffering, reduce treatment cost, and achieve a better clinical prognosis.

Keywords: preauricular fistula, local flap, infection, surgery, treatment

\section{Introduction}

Congenital preauricular fistula is a common congenital ear deformity in otolaryngology. It can occur with one or both ears and originates either from the formation of the first and second branchial arches or from the first branchial clefts of the auricle. According to the different opening positions of the preauricular fistula, it can be divided into common type (opens in the front area of the ascending helix branch) and variant type (opens in the area behind the imaginary line of the tragus and the rear edge of the ascending helix branch). ${ }^{1}$ The incidence of common preauricular fistulas is higher than that of the variant. By clinical observations, after an infection occurs, the chance of recurring infection will become extremely high. $^{2-4}$ To stop infection reoccuring, a best strategy is to completely remove the fistula. But there is controversy of the time point to perform the preauricular fistula 
removing surgery. Following the traditional standard, the surgery must be performed after full control of the infection. ${ }^{5}$ However, we found that the infection is hard to be effectively controlled by conventional infection treatment (giving antibiotics, repeated incision, drainage, etc), and such a process leads to a very long treatment period, increasing medical expenses and mental burden on patients. In most recently studies and clinical observations, more clinicians advocated that the preauricular fistulas resection surgery should be performed as early as possible, even along with the infection, to achieve a better prognosis. ${ }^{6}$

In this study, we successfully performed the congenital preauricular fistula resection during infection period by combining local flap repairing. The biggest challenge of our surgery is that the scope of surgical resection is larger than the conventional surgery in order to completely remove both lesion tissue and infection tissue around the fistula. In this situation, whether the surgical cavity can be closed smoothly is a key factor hindering clinicians' daring to perform such operations. From previous studies, some clinicians try to perform forced sutures for large local tissue defects, but it usually resulted in delayed wound healing, auricle deformity, excessive scar proliferation, and even changes in the external canthus shape. Alternatively, there is a worry that tissue defects cannot be effectively repaired as the scope of resection is larger, those trying will still lead to an increase in the recurrence rate, ending in a prolonged treatment period and increased medical cost. We are the first here to introduce local flap to repair large surgical cavities of the preauricular fistula removing surgery, the duration of treatment, healing index, and total cost between our local flaps combining surgery treatment and conventional treatment of preauricular fistula were evaluated. By detailed reporting of the strategy of our treatment for congenital preauricular fistula, we wish this complete resection combining with local skin flap surgery method can be widely introduced into the treatment of preauricular fistula to largely reduce the patient's suffering.

\section{Materials and Methods}

The study was conducted in accordance with the Declaration of Helsinki and was approved by the Wuhan No. 1. Hospital and Ningbo No. 3 Hospital ethics institutional committees, Hubei and Zhejiang Province, China. All subjects gave their written informed consent.

\section{Diagnosis}

The diagnosis of congenital preauricular fistula mainly depends on medical history and physical examination in this study. A sinus pit in a typical site (usually found from birth) is highly suggested in the diagnosis. Erythema, swelling, pain, and discharge are common signs and symptoms of infection. Ultrasonography is applied to rule out the congenital preauricular fistula as part of Branchio-OtoRenal (BOR) syndrome or Trisomy from kidney disease.

\section{Clinical Cases}

In total, 100 cases were collected from January 2016 to March 2020, 50 cases belonged to the conventional treatment group and the other 50 cases were in the local flap repairing group. All selected cases were drawn from two medical centers and classified into two groups according to the surgical methods performed. Inclusion criteria of patients: 1) diagnosed as common type preauricular fistula; 2) had single ear infections; 3) age $\geq 18$ years; and 4) need further lesion remove surgery. Exclusion criteria: Patients were diagnosed with common type congenital preauricular fistula, but did not complete the whole treatment process set in this study. The age, gender, and lesion side distribution of included patients are shown in Table 1.

\section{Treatment Groups}

In the conventional treatment group, the treatment timeline starts with conventional infection control. These infection control treatments include but are not limited to antiinflammation (by giving antibiotics), lesion incision and drainage, dressing change, and so on. Once the infection of the patients was totally treated, the patients had the preauricular fistula removing surgery. In the local flap repairing group, the treatment skips the whole conventional infection control process. Some included patients may

Table I Age, Gender and Lesion Side Distribution of Included Patients

\begin{tabular}{|c|c|c|c|c|}
\hline & & $\begin{array}{c}\text { Flap Repairing } \\
\text { Treatment }\end{array}$ & $\begin{array}{l}\text { Conventional } \\
\text { Treatment }\end{array}$ & $p$ \\
\hline Patient & & 50 & 50 & \\
\hline Age (yea & & $34.34 \pm 12.07$ & $36.74 \pm 10.99$ & 0.301 \\
\hline Gender & Male & 37 (74\%) & 35 (70\%) & 0.656 \\
\hline & Female & $13(26 \%)$ & $15(30 \%)$ & \\
\hline Lesion & Right & 21 (42\%) & $19(38 \%)$ & 0.683 \\
\hline side & Left & 29 (58\%) & 31 (62\%) & 0.683 \\
\hline
\end{tabular}


take lesion incision and drainage just once, then the patients will have the preauricular fistula removal combined with local flap repairing surgery directly, regardless of the infection.

The surgery operation criteria are as follows: Conventional treatment group: 1) All patients had a common preauricular fistula with a fistula located in front of the ear, 2) The patients had undergone multiple outpatient incisions and drainage of pus or took antibiotics, and their cases were effectively controlled, and 3) The patients could tolerate general anesthesia in the preoperative examination; Local flap repairing group: 1) All patients had a preauricular fistula during the common infection stage, with the fistula located in front of the ear, 2) The patients had experienced at least one outpatient incision and drainage in the outpatient department, 3) The infection around the fistula was visible, 4) The skin of the focus was swollen and damaged, and there was granulation tissue hyperplasia, necrotic tissue formation, or local skin swelling, and 5) The patients could tolerate general anesthesia in the pre-operative examination. All patients signed their informed consent.

\section{Surgical Process}

All patients underwent oral intubation and general anesthesia. All procedures were performed by doctors in the same medical group (the doctors had the same experience in surgical training), and routine skin preparations were performed before the operation. The main surgery range was approximately $5 \mathrm{~cm}$ from the hairline.

Patients in the conventional treatment group received a fusiform incision around the fistula. Guided by the probe, the tissues were separated along the fistula until the fistula was completely resected. If necessary, a microscope was used to find the fistula and its branches. Therefore, the surgical cavity of such patients was generally small, so it could be stitched together straight.

For patients in the local flap repairing group, a large fusiform incision was made on the normal skin approximately $2 \mathrm{~mm}$ from the periphery of the infected foci. The fistula and the infected tissue had to be included in the incision. The skin and subcutaneous tissue were cut by using ophthalmic scissors or the skin and subcutaneous tissue were cut layer by layer and separated to the deep part until the superficial temporal fascia was exposed, and this was used as the base of the resection. Along the surface of the superficial temporal fascia, according to the scope of the infection, the infection and necrotic tissue were gradually separated and removed until there was no visible necrotic tissue or infection foci in the entire surgical cavity. If the infected foci invaded the tragus cartilage, part of the tragus cartilage was removed. As the infection foci of this type are generally located in front of and below the fistula, after complete removal of the infected foci and necrotic tissue, the surgical cavity defect had a roughly (irregularly) round shape. For post-operative defects in the surgical area, the following treatment methods were adopted: 1) If the diameter of the defect in the surgical area was less than $1 \mathrm{~cm}$, the surgical cavity was directly sutured. 2) If the diameter of the defect was greater than $1 \mathrm{~cm}$, the local flap from the posterior auricular transferred to the frontal of ear to cover the surgical defect, or the Burow wedge flap was used to repair the defect of the surgical ear. Case examples for those specific operations are shown in the Results section.

\section{Statistical Analysis}

SPSS 26.0 statistical software was used to process the data statistical analysis. Measurement data obeying a normal distribution was expressed as the Mean \pm standard deviation (SD), and an independent sample $t$-test was used for comparison between groups. The measurement data did not obey a normal distribution, represented by the median (P25, P75), and two independent Mann-Whitney $U$-tests were used for comparison between groups. The enumeration data was expressed as the frequency and rate (\%), and the comparison between groups was performed by $\chi^{2}$ or $Z$-test. $P<0.05$ indicates that the difference is statistically significant. Photos of the surgery operation were taken manually using a cellphone camera (Leica, 48 million pixels) during the operation.

\section{Results}

\section{The Age, Gender, and Lesion Side Distribution of Included Patients}

The age, gender, and lesion side of patients in the conventional treatment group and the pedicled flap repairing treatment group conformed to the normal distribution. There was no statistically significant difference of age, gender, or lesion side distribution between two groups ( $t$-test, $p>0.05$ ) (Table 1).

\section{Case Examples}

In Figure 1, we showed a case example of the posterior auricular skin flap repairing operation: The local flap was 

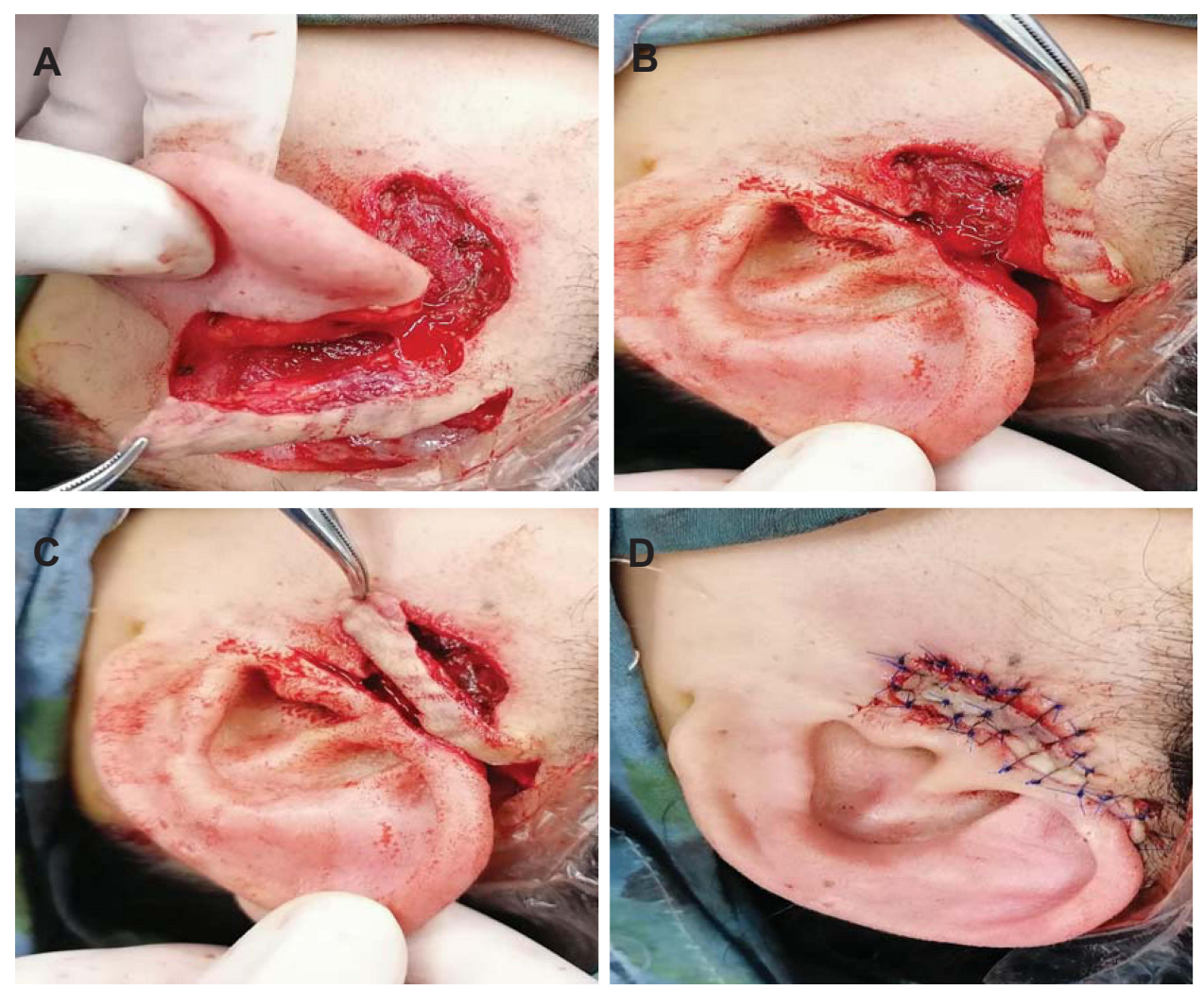

Figure I (A) Posterior auricular skin flap preparation; (B) a transfer of the posterior auricular skin flap to the front of the ear; (C) repair of the preauricular defect with the posterior auricular skin flap; (D) surgical site appearance after the flap suture.

transplanted from the posterior auricular to repair anterior defects. In this case, a posterior auricular flap was prepared after resection of the preauricular fistula and infected tissue (shown in Figure 1A), the size of the flap must be able to completely cover the preauricular defect and to ensure that there is no tension during suture. The blood supply of the flap could not be damaged. Then, we rotated the flap from the back of the ear to the front of the ear to cover the defect. During the rotation, the bottom of the flap must be carefully treated to avoid any twisting, which would damage the blood supply of the flap (shown in Figure 1B). The next operation is to cover the defect using the prepared flap, the bottom of the flap will be sutured to exclude any possible dead space (shown in Figure 1C). At the end, the flap could be sutured as shown in Figure 1D.

We show another case example of the Burow wedge flap repairing in Figure 2. First, we labeled out a preoperative incision (Figure 2A), the right part of the incision must include the preauricular fistula and infection tissue. Second, we completely resected the preauricular fistula and all the infection tissue (Figure 2B). Then, preparing a Burow wedge flap as shown in Figure $2 \mathrm{C}$, during this procedure, the same, the blood supply of the flap must be carefully protected as well as avoiding any damage to the parotid fascia. At the end, we sutured the flap as shown in Figure 2D, and the dead space, which will drive the fluid accumulation, must be avoided to lower the infection risk.

\section{Preauricular Fistula Resecting Combining Local Flap Repairing Surgery Operation Shortened the Treatment Period and Reduced the Treatment Cost}

The total duration of treatment was defined as the period from any first treatment starting day to the day of patient discharge (in days). After the surgery operation, it usually took about $7 \pm 2$ days to remove the sutures in the conventional treatment group and $7 \pm 2.5$ days in the local flap repairing group, there was no significant difference of this post-operative recovery period between the two groups ( $t$-test, $p>0.05)$. However, in our study, the total duration of treatment in the two groups was largely different, it took $43.06 \pm 8.24$ days in the conventional treatment group 

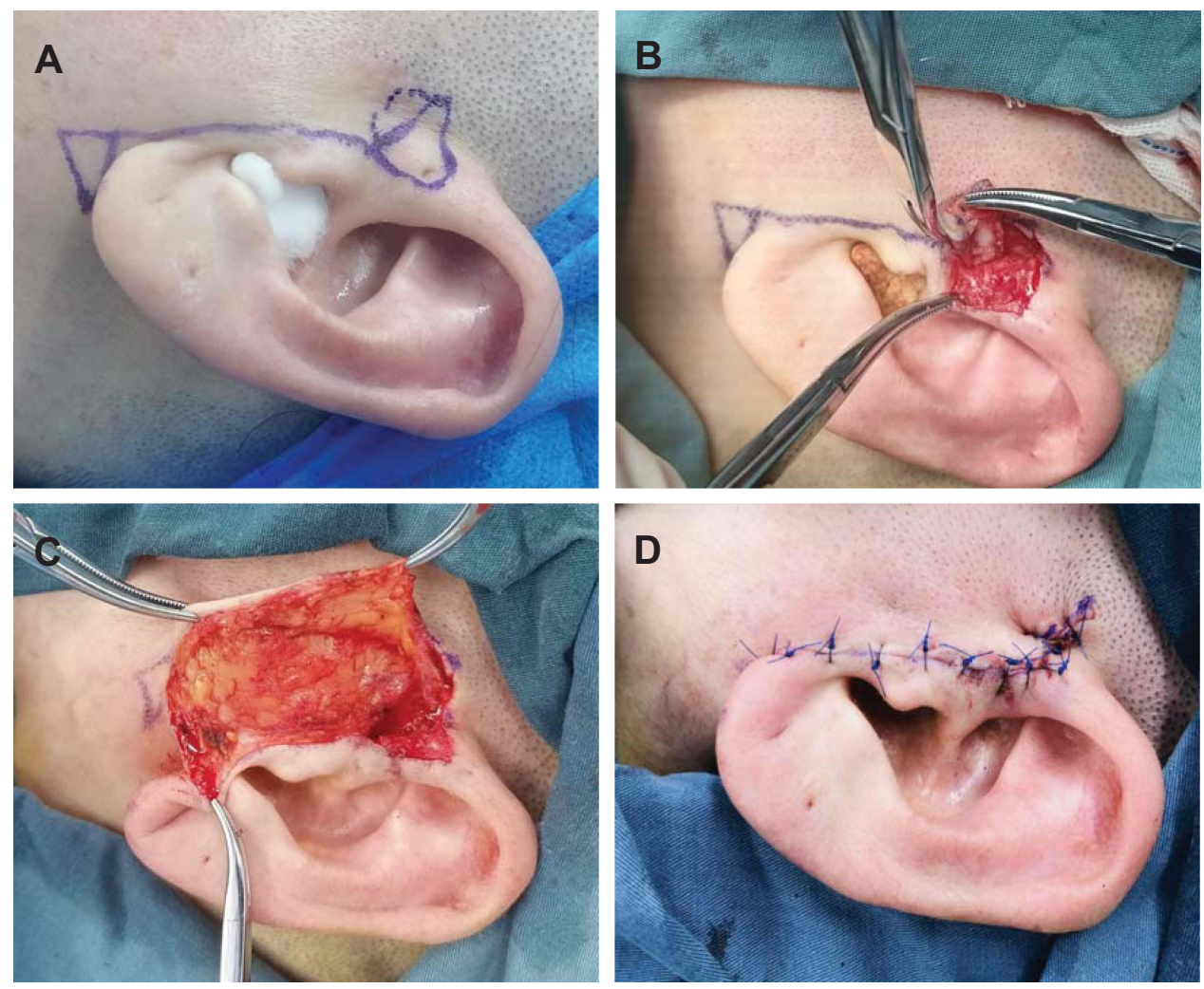

Figure 2 (A) Pre-operation label of the surgical incision; (B) resection of the preauricular lesion; (C) preparation of the Burow flap; (D) surgical site appearance after the preauricular incision is sutured.

$(n=50)$, but only $13.98 \pm 2.14$ days in the local flap repairing group $(n=50)$, the total treatment period was significantly shortened in the local flap repairing group by performing our improved treatment of preauricular fistula $(t$-test, $p<0.05)$ (Table 2). We would say that this causes a large relief of patient's suffering from the preauricular fistula.

Meanwhile, we also analyzed the total cost in conventional treatment and local flap repairing treatment groups. For each case, the total cost analysis included outpatient cost and inpatient cost analysis. In addition, we also decomposed the outpatient cost and inpatient cost of

Table 2 Treatment Duration and Total Cost

\begin{tabular}{|l|c|c|c|}
\hline & Flap Repairing & Conventional & $\boldsymbol{p}$ \\
\hline $\begin{array}{l}\text { Treatment } \\
\text { duration } \\
\text { (Days) }\end{array}$ & $13.98 \pm 2.14$ & $43.06 \pm 8.24$ & $<0.000 \mathrm{I}$ \\
\hline Total cost/ & $7,068.03$ & $15,004.98$ & $<0.000 \mathrm{I}$ \\
patient (CNY) & $(6,338.91$, & $\begin{array}{l}(13,007.23, \\
16,290.89)\end{array}$ & \\
& $8,044.58)$ & 16,890 & \\
\hline
\end{tabular}

each case into medicine cost and treatment cost. As the total cost did not conform to a normal distribution in both two groups, the median value of the cost was used in the following: the total cost was $¥ 7,068.03$ per patient (in CNY) in the flap repairing treatment group, and was as high as $¥ 15,004.98$ per patient in the conventional treatment group. By taking our improved surgery treatment, the total cost was reduced by $52.3 \%$ of that with the conventional treatment method ( $t$-test, $p<0.05$ ) (Table 2). A detailed further analysis of the spending pathway is shown in Table 3, there are significant differences in the outpatient medicine cost, outpatient treatment cost, inpatient treatment cost, but no significant difference in the inpatient medicine cost between the two groups.

\section{Post-Operation Complications}

In terms of post-operative complications, we mainly observed wound infection and scarring in our study.

In the conventional treatment group, two patients had wound infections, and one patient had hyperproliferation of scars. In the local flap repairing group, four patients showed post-operative wound infections, and two patients had hyperproliferation of scars. The difference was not 
Table 3 Analysis of Outpatient Medicine/Treatment Cost and Inpatient Medicine/Treatment Cost in Conventional and Local Flap Repairing Treatment Groups

\begin{tabular}{|l|c|c|c|}
\hline & Flap Repairing & Conventional & $\boldsymbol{P}$ \\
\hline Outpatient medicine cost (CNY) & $143.11 \pm 31.30$ & $712.14 \pm 221.74$ & $<0.0001$ \\
Outpatient treatment cost (CNY) & $214.28 \pm 52.93$ & $2,631.28 \pm 665.45$ & $<0.0001$ \\
Inpatient medicine cost (CNY) & $1,241.18 \pm 54.79$ & $1,334.45 \pm 345.28$ & 0.065 \\
Inpatient treatment cost (CNY) & $5,518.75$ & $10,227.29$ & $<0.0001$ \\
\hline
\end{tabular}

Table 4 Post-Operation Complications

\begin{tabular}{|l|c|c|c|}
\hline & Flap Repairing & Conventional & $\boldsymbol{p}$ \\
\hline Wound infection & $4(8 \%)$ & $2(4 \%)$ & 0.678 \\
Scarring & $2(4 \%)$ & $1(2 \%)$ & $>0.9999$ \\
\hline
\end{tabular}

statistically significant between groups $(t$-test, $p>0.05)$ (Table 4).

Detailed reports of those patients with post-operative complications are as follows: 1) Wound infection complication: In the local flap repairing group, there were four patients who showed wound redness and swelling in appearance, with clear exudates seen after the operation. Accordingly, we changed the dressing, extended the antibiotic time, and delayed the suture removal for them. From their hospital history, we found that two patients had diabetes, one patient had a defect with a diameter greater than $2.5 \mathrm{~cm}$, and one patient had poor blood supply to the flap. In the conventional treatment group, two patients had wound redness and swelling. We performed conventional post-operative dressing change, prolonged antibiotic use, and delayed suture removal. We did not find other related diseases from their histories. 2) Scarring: After suture removing, the edge of the flap for most patients was bluish-purple color, there was no obvious scar hyperplasia and no changes in the appearance of the auricle and lateral canthus. However, two patients in the local flap repairing group and one patient in the conventional treatment group showed a scar constitution. We did not take action on the scarring complication.

\section{Discussion}

Resection of a congenital preauricular fistula is a common operation in otolaryngology. The standard technique is to excise an ellipse of skin surrounding the preauricular sinus opening and to resect the individual tract: the simple sinectomy. A supra-auricular approach was first made by Prasad et al in 1990, which is still widely be used until now, and there are also many improved techniques based on it. Another common operation is the inside-out technique, which was first introduced by Jesma in Rotterdam..$^{7-9}$ With the gradual in-depth understanding of its genesis, anatomy, and surgical concepts, the post-operative recurrence rate has been declining, but still holds a level around $6.1-10 \% .^{3}$ There is always a controversy about whether to perform surgery during the infection period. ${ }^{10}$ Some clinicians believe that surgery during the infection period will increase the chance of recurrence. ${ }^{11}$ Recently, an increasing number of studies have gradually paid attention to the surgical treatment of congenital preauricular fistulas during infection. $^{6,12}$

In this study, we designed a comparison between conventional treatment of the preauricular fistula removing and improved surgery of preauricular fistula removing combining with local flap repairing. Our team found that preauricular fistula resection surgery combining with local flap repairing can be performed even during the infection period, and our improved surgery can not only reduce the frequency and pain of excision and drainage of pus of the patients, but also shorten the treatment duration of the entire disease and reduce medical costs.

There are some reports talking about the surgical rules for the resection of congenital preauricular fistulas during infection. As a summary, the points are described as follows: 1) Surgery can be performed as early as possible; ${ }^{13}$ 2) Based on the location of the fistula and the scope of infection, operation combined with anatomical markers or a complete resection along the superficial surface of the temporalis can be used; ${ }^{14}$ and 3) Resected tissues can involve infected necrotic tissue, granulation, and even cartilage. ${ }^{15}$ In our study, by modifying the surgical skills in previous reports, adopting the protection of important structures, our team introduced a new surgery strategy of completely removing the infected foci and all necrotic tissues in the treatment of preauricular fistulas. If it is 
difficult to identify the foci with the naked eye during the operation, a microscope was used to help determine the scope of infection. In response to some scholars' questions regarding whether surgery can complete the removal of the fistula during the infection period and whether surgery during the infection period will lead to the possibility of the spread of infection, we suggest the following: 1) During surgery in the infection period, the infected area must be larger than the area where the fistula grows. Therefore, as the necrotic tissue and the infected foci are completely removed during the operation, the preauricular fistula and its branches must have been completely removed. 2) The experimental group cases underwent at least one incision and drainage in the outpatient clinic. The scope of infection was limited. There was no diffuse redness or swelling on the affected side. The skin around the infected area was normal, the skin temperature was not high, and there was no tenderness. Patients in this period should be in a quiescent period of infection observed by our research team. None of the 50 enrolled patients had postoperative spread of infection in this study.

We noted the history of the patients in the local flap treatment group who have wound healing delay in the Results section. By a further analysis, the main reasons which affected the wound delay come from: 1) diabetes, 2) The area of infection and necrotic tissue removed was large, and 3) The blood supply of the flap was damaged when the local flap was cut.

Regarding the criteria to have the flap repairing surgery, we suggest: 1) Defects of approximately $1 \mathrm{~cm}$ in diameter can be directly sutured; 2) For defects larger than $1 \mathrm{~cm}$, pedicled flaps are required. Overall, the surgeon's experience is also a main determinant.

In order to reduce the necrosis rate of the skin flaps after surgery, the surgeons should choose a familiar flap operation, and be cautious to protect the blood supply of the skin flaps during the surgery. In addition, attention should be paid to avoid any dead space during any sealing or suturing process. Due to the abundant facial vascular network within the operation scope, necrosis of the pedicle flap usually will not show obvioussigns during the operation. To avoid this happening, here are some tips: 1) The aspect ratio of pedicled flaps is generally smaller than 1.5:1, and 2) For the Burow flap preparation, the separation could be performed along the fascial surface of the parotid gland to avoid damage to the vascular perforation.

The posterior auricular flap and the Burow wedge flap are generally used to repair the defects caused by resection of the malignant tumor or trauma in the head and neck. ${ }^{16-18}$ In this study, we introduced the two local flaps to repair the defects after resection of the preauricular fistula and infection tissue. Based on the results of our study, we strongly recommend that the preauricular fistula should be removed even during its infection period, a successful surgery treatment can be achieved by complete resection of the preauricular fistula and infection tissues and combining with the local skin flap repairing. The introduced flap improved method can effectively repair the preauricular tissue defect and avoid complications such as excessive scar hyperplasia, auricle deformity, and lateral canthal deformation caused by excessive defects and direct sutures. Limited to the case number in the study, it is necessary to conduct a larger sample study in the future to arrive at a better standard treatment plan.

\section{Acknowledgments}

We gratefully acknowledge Dr. Shouguo Yao for providing a scientific guide to this research.

\section{Author Contributions}

All authors made a significant contribution to the work reported, whether that is in the conception, study design, execution, acquisition of data, analysis and interpretation, or in all these areas; took part in drafting, revising, or critically reviewing the article; gave final approval of the version to be published; have agreed on the journal to which the article has been submitted; and agree to be accountable for all aspects of the work.

\section{Funding}

This work was supported by Ningbo Medical and Health Science Plan, No. 2019ZD013.

\section{Disclosure}

The authors have no conflicts of interest to report.

\section{References}

1. Kim JR, Kim DH, Kong SK, et al. Congenital periauricular fistulas: possible variants of the preauricular sinus. Int $J$ Pediatr Otorhinolaryngol. 2014;78(11):1843-1848. doi:10.1016/j. ijporl.2014.08.005

2. Choi SJ, Choung YH, Park K, Bae J, Park HY. The variant type of preauricular sinus: postauricular sinus. Laryngoscope. 2007;117 (10):1798-1802. doi:10.1097/MLG.0b013e3180caa1ca

3. El-Anwar MW, ElAassar AS. Supra-auricular versus Sinusectomy Approaches for Preauricular Sinuses. Int Arch Otorhinolaryngol. 2016;20(4):390-393. doi:10.1055/s-0036-1583305 
4. Pang J, Xiong H, Liao Q, Xu Y, Huang Q, Ou Y. The diagnosis and treatment of a variant type of auricular sinus: postauricular sinus. Eur Arch Otorhinolaryngol. 2019;276(7):1961-1967. doi:10.1007/ s00405-019-05431-7

5. Das C, Khaowas A. Pre-auricular sinus with post-auricular extension: an uncommon variant. Indian J Otolaryngol Head Neck Surg. 2019;71(Suppl 2):1511-1514. doi:10.1007/s12070-019-01605-1

6. Han JS, Park JM, Han JJ, et al. Surgical results of infected preauricular sinus: no need for delay. Int $J$ Pediatr Otorhinolaryngol. 2020;135:110129. doi:10.1016/j.ijporl.2020.110129

7. Huang WJ, Chu CH, Wang MC, Kuo CL, Shiao AS. Decision making in the choice of surgical management for preauricular sinuses with different severities. Otolaryngol Head Neck Surg. 2013;148 (6):959-964. doi:10.1177/0194599813483447

8. Baatenburg de Jong RJ. A new surgical technique for treatment of preauricular sinus. Surgery. 2005;137(5):567-570. doi:10.1016/j. surg.2005.01.009

9. Khardali MH, Han JS, Kim SI, et al. Clinical efficacy of standard simple elliptical incision following drain-less and subcutaneous suture technique in preauricular sinus surgery. Am J Otolaryngol. 2020;41(4):102465. doi:10.1016/j.amjoto.2020.102465

10. Isaacson G. Comprehensive management of infected preauricular sinuses/cysts. Int J Pediatr Otorhinolaryngol. 2019;127:109682. doi:10.1016/j.ijporl.2019.109682

11. Rataiczak H, Lavin J, Levy M, Bedwell J, Preciado D, Reilly BK. Association of recurrence of infected congenital preauricular cysts following incision and drainage vs fine-needle aspiration or antibiotic treatment: a retrospective review of treatment options. JAMA Otolaryngol Head Neck Surg. 2017;143(2):131-134. doi:10.1001/ jamaoto.2016.2988
12. Wang L, Wei L, Lu W, Liu Z, Wang M, Wang Z. Excision of preauricular sinus with abscess drainage in children. Am J Otolaryngol. 2019;40(2):257-259. doi:10.1016/j.amjoto.2018. 10.016

13. Choo OS, Kim T, Jang JH, Choung YH. The clinical efficacy of early intervention for infected preauricular sinus. Int $J$ Pediatr Otorhinolaryngol. 2017;95:45-50. doi:10.1016/j.ijporl.2017.01.037

14. Nagalingeswaran A, Kumar RD. Infra-auricular Sinus: a Very Rare Case Presentation. Indian J Otolaryngol Head Neck Surg. 2019;71 (Suppl 2):1502-1504. doi:10.1007/s12070-019-01636-8

15. Kim WJ, Lee YM, Kim DH, et al. Causes and prevention of revision surgery for preauricular sinus: a histopathological analysis. Int J Pediatr Otorhinolaryngol. 2019;116:199-203. doi:10.1016/j. ijporl.2018.11.006

16. Quatrano NA, Dawli TB, Park AJ, Samie FH. Simplifying Forehead Reconstruction: a Review of More Than 200 Cases. Facial Plast Surg. 2016;32(3):309-314. doi:10.1055/s-0036-1579780

17. Goulao J. Reconstruction of a large nasal defect. J Dermatolog Treat. 2014;25(5):444-445. doi:10.3109/09546634.2012.755257

18. Portilla N, Cerci FB, Tolkachjov SN. Hinge flaps with Burow's grafts for reconstruction of deep facial defects. J Am Acad Dermatol. 2020. doi:10.1016/j.jaad.2020.04.100
International Journal of General Medicine

\section{Publish your work in this journal}

The International Journal of General Medicine is an international, peer-reviewed open-access journal that focuses on general and internal medicine, pathogenesis, epidemiology, diagnosis, monitoring and treatment protocols. The journal is characterized by the rapid reporting of reviews, original research and clinical studies

\section{Dovepress}

across all disease areas. The manuscript management system is completely online and includes a very quick and fair peer-review system, which is all easy to use. Visit http://www.dovepress.com/ testimonials.php to read real quotes from published authors. 